Swarthmore College

Works

12-1-1985

\title{
Polarization Asymmetry In The Photodisintegration Of The Deuteron
}

\author{
M. P. De Pascale \\ G. Giordano \\ G. Matone \\ D. Babusci \\ R. Bernabei
}

See next page for additional authors

Follow this and additional works at: https://works.swarthmore.edu/fac-physics

Part of the Physics Commons

Let us know how access to these works benefits you

\section{Recommended Citation}

M. P. De Pascale, G. Giordano, G. Matone, D. Babusci, R. Bernabei, Oleksa-Myron Bilaniuk, L. Casano, S. d'Angelo, M. Mattioli, P. Picozza, D. Prosperi, C. Schaerf, S. Frullani, and B. Girolami. (1985). "Polarization Asymmetry In The Photodisintegration Of The Deuteron". Physical Review C. Volume 32, Issue 6.

1830-1841. DOI: 10.1103/PhysRevC.32.1830

https://works.swarthmore.edu/fac-physics/346

This work is brought to you for free by Swarthmore College Libraries' Works. It has been accepted for inclusion in Physics \& Astronomy Faculty Works by an authorized administrator of Works. For more information, please contact myworks@swarthmore.edu. 


\section{Authors}

M. P. De Pascale, G. Giordano, G. Matone, D. Babusci, R. Bernabei, Oleksa-Myron Bilaniuk, L. Casano, S. d'Angelo, M. Mattioli, P. Picozza, D. Prosperi, C. Schaerf, S. Frullani, and B. Girolami 


\title{
Polarization asymmetry in the photodisintegration of the deuteron
}

\author{
M. P. De Pascale, G. Giordano, and G. Matone \\ Istituto Nazionale di Fisica Nucleare, Laboratori Nazionali di Frascati, Frascati, Italy
}

D. Babusci, R. Bernabei, O. M. Bilaniuk, L. Casano, S. d'Angelo, M. Mattioli, P. Picozza, D. Prosperi, and C. Schaerf

Dipartimenti di Fisica, Università di Roma, Roma, Italy and Istituto Nazionale di Fisica Nucleare, Sezione di Roma, Roma, Italy

\author{
S. Frullani and B. Girolami \\ Istituto Superiore di Sanità, Roma, Italy and Istituto Nazionale di Fisica Nucleare, Sezione Sanità, Roma, Italy
}

(Received 12 August 1985)

\begin{abstract}
The reaction ${ }^{2} \mathbf{H}(\gamma, p)$ n has been studied using a monochromatic and polarized gamma ray beam at energies $E_{\gamma}=19.8,29.0,38.6$, and $60.8 \mathrm{MeV}$. The beam of an intensity $\sim 4 \times 10^{5} \gamma / \mathrm{sec}$ was obtained by Compton back scattering of mode-locked laser light off electron bunches in the Adone storage ring. Photoneutron yields were measured at nine neutron angles $\theta_{\mathrm{n}} \simeq 15,30,45,60,90,120$, 135,150 , and $165 \mathrm{deg}$ in the center of mass (c.m.) for $E_{\gamma}=19.8,29.0$, and $38.6 \mathrm{MeV}$, and at $\theta_{\mathrm{n}} \simeq 30$, $60,90,120$, and $150 \mathrm{deg}$ c.m. for $E_{\gamma}=60.8 \mathrm{MeV}$. The polarization independent component $I_{0}(\theta)$ of the differential cross section and the polarization dependent component $P I_{1}(\theta)$ were deduced and the angular distribution of the azimuthal asymmetry factor $\Sigma(\theta)=I_{1}(\theta) / I_{0}(\theta)$ was obtained. An extensive comparison with theory has been carried out and the inclusion of corrections due to meson exchange currents and to $\Delta$-isobar configurations have been shown to be mandatory at energies $E_{\gamma} \geq 40 \mathrm{MeV}$. Theoretical and experimental implications of intermediate energy deuteron photodisintegration studies are discussed in some detail.
\end{abstract}

\section{INTRODUCTION}

The deuteron photodisintegration ${ }^{2} \mathbf{H}(\gamma, \mathrm{p}) \mathrm{n}$ is a basic nuclear process, and as such it has been extensively used for studies of electromagnetic properties of nucleons and of the nucleon-nucleon $(\mathrm{N}-\mathrm{N})$ interactions. As of recently, the development of advanced computational capabilities and of new experimental techniques has thrust the ${ }^{2} \mathbf{H}(\gamma, p) \mathbf{n}$ reaction into the forefront of renewed experimental and theoretical interest.

In particular, it has become evident that non-nucleonic. degrees of freedom play an essential role in the dynamics of the deuteron breakup at intermediate energies. Because of their relatively long range, the $\pi$-meson exchange currents $(\pi$-MEC) are of prime importance, but as the $\gamma$ ray energy increases, the subnucleonic degrees of freedom due to internal excitation of the isobar configurations (IC) are expected to come into play.

A number of refined calculations of the deuteron photodisintegration cross section below the pion production threshold have become available in the last few years. ${ }^{1-25}$ Among other things, these calculations indicate the importance of mesonic degrees of freedom within the deuteron at gamma energies as low as 30 or $40 \mathrm{MeV}$. The experimental implications of these calculations point to a need for new cross section measurements using monochromatic and polarized gamma beams in the energy region below the pion threshold, a range for which so far very few data are available. Particularly important are the measurements of the polarization independent part of the differential cross section $I_{0}(\theta)$, of the polarization dependent part $P I_{1}(\theta)$, and of the angular distribution of

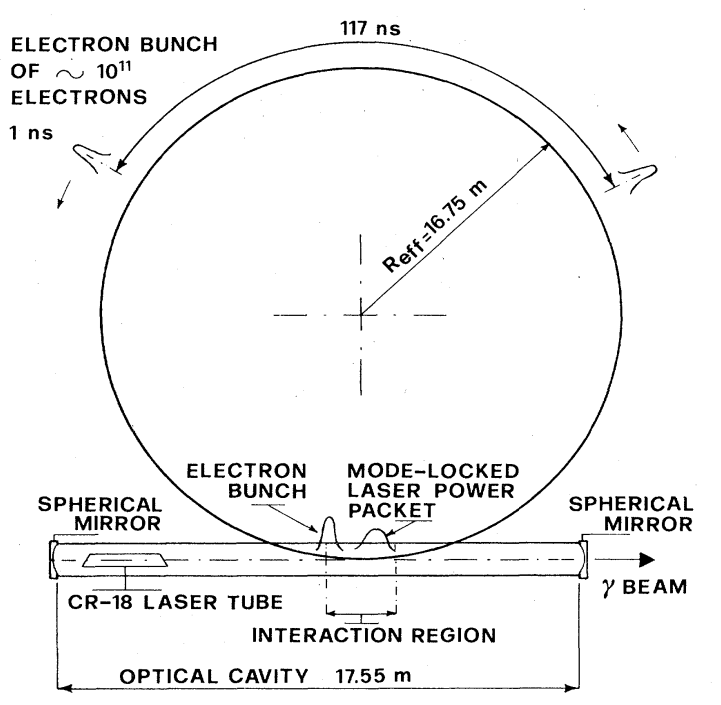

FIG. 1. Schematic representation of the LADON process (not to scale). The effective circumference (i.e., the perimeter consisting of circular and straight sections) is $2 \pi R_{\text {eff }}=105.3 \mathrm{~m}$, so that a bunch of ultrarelativistic electrons takes about $351 \mathrm{nsec}$ to make the round. Under normal operation there are three such bunches circulating in the ring, so that they succeed one another every $117 \mathrm{nsec}$. This time interval defines the length $L=17.55 \mathrm{~m}$ of the optical cavity in which a laser power packet bounces back and forth between the cavity end mirrors. Compton back scattering of the laser photons off the electrons takes place in a well defined portion of a straight segment of the storage ring free from magnetic field (Refs. 26, 27, and 32). 
TABLE I. Intensity (in units of $10^{5}$ gammas/sec), resolution (\%), and linear polarization degree $P$ of the LADON beam at the $\gamma$ energies of our experiment.

\begin{tabular}{cccc}
\hline \hline $\begin{array}{c}E_{\gamma} \\
(\mathrm{MeV})\end{array}$ & $\begin{array}{c}\gamma / \mathrm{sec} \\
\left(10^{5}\right)\end{array}$ & $\begin{array}{c}\Delta E / E \\
(\%)\end{array}$ & $P$ \\
\hline 19.8 & 1.0 & 3.00 & 0.999 \\
29.0 & 1.1 & 5.00 & 0.998 \\
38.6 & 1.3 & 5.74 & 0.997 \\
60.8 & 1.8 & 7.55 & 0.994 \\
\hline \hline
\end{tabular}

the azimuthal asymmetry factor $\Sigma(\theta)=I_{1}(\theta) / I_{0}(\theta)$ (see Sec. III) which turns out to be remarkably sensitive to meson exchange currents and to virtual excitation of nucleonic $\Delta$-isobar configurations.

The present work constitutes the first stage of a more extensive research program that addresses this need. The source of $\gamma$ rays, developed at Frascati and named LADON, ${ }^{26,27}$ is laser light Compton back-scattered off ultrarelativistic electrons circulating in the Adone storage ring.

Besides the LADON project, there have been to date three major experimental efforts that resulted in Compton back-scattered photon beams (Lebedev Institute, ${ }^{28}$ Cambridge, ${ }^{29}$ and Stanford $\left.{ }^{30}\right)$. Because in all three cases an external laser beam has been used, the resulting gamma beam intensity was very low and only the Stanford beam had been used in an actual experiment. ${ }^{30}$ On the other hand, in the LADON process the Compton scattering takes place within an optical cavity that is superposed onto a straight section of the storage ring and where the interaction takes place between compact bunches of electrons and phase-locked traveling pulses of concentrated laser power (see Sec. II). This "photon bunching" and the use of a storage ring are the two most important aspects of the LADON approach (see Fig. 1). After some stages of development, the LADON facility can now provide gamma ray beams of an intensity of $(3-5) \times 10^{5}$

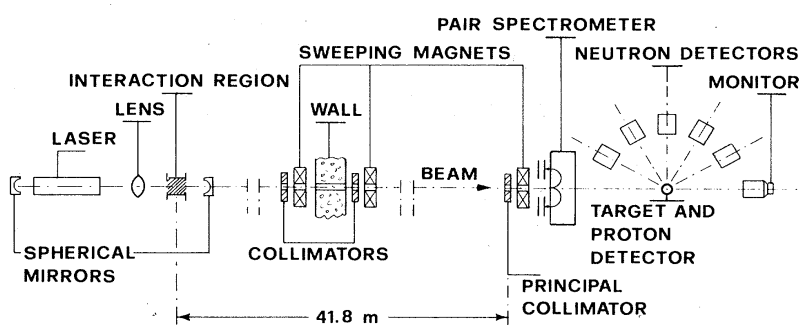

FIG. 2. Layout of the experimental area. The laser beam is aligned to $10 \mu \mathrm{m}$ by on-line remote control via CAMAC using a PDP 11/04 minicomputer and position transducers. Three sweeping magnets clear the gamma beam of all charged particles. The principal collimator restricts the beam to a solid angle of $2.5 \times 10^{-8} \mathrm{sr}$. The energy profile of the gamma beam is continuously monitored by a magnetic pair spectrometer. A cylinder of deuterated scintillation liquid NE-230 serves both as target and proton detector. Neutron time of flight spectra are recorded at five angles using NE-213 scintillators and pulseshape analysis.

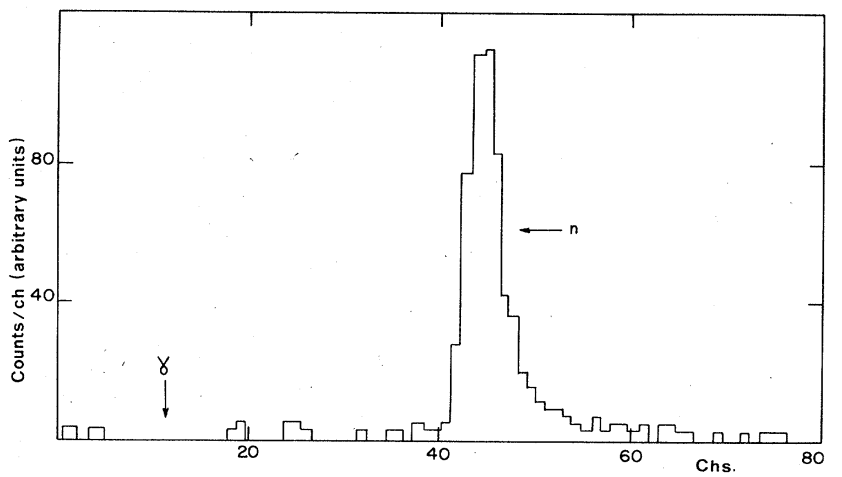

FIG. 3. A typical time of flight spectrum at $E_{\gamma}=19.8 \mathrm{MeV}$ ( $0.8 \mathrm{nsec}$ per channel), obtained after background reduction by pulse shape discrimination and rejection of events that fall outside of the kinematic limits for the proton energy. The neutron peak $\mathrm{n}$ is clearly shown; the window indicated by the $\gamma$ symbol corresponds to the region of the $\gamma$ background, cut out by a hardware system.

photons/sec. This has permitted the accumulation of a considerable amount of new data on deuteron photodisintegration, particularly on the azimuthal asymmetry.

Reported here are measurements of photoneutron yields, carried out at nine c.m. neutron angles $\left(\theta_{\mathrm{n}} \simeq 15,30\right.$, $45,60,90,120,135,150$, and $165 \mathrm{deg}$ ), for three gamma energies $\left(E_{\gamma}=19.8,29.0\right.$, and $\left.38.6 \mathrm{MeV}\right)$ and at $\theta_{\mathrm{n}} \simeq 30$, $60,90,120$, and $150 \mathrm{deg}$ for $E_{\gamma}=60.8 \mathrm{MeV}$. Each set of measurements consisted of a series of runs, with the angle $\varphi$ between the reaction plane and the gamma polarization plane alternating repeatedly between $\varphi=0^{\circ}$ and $\varphi=90^{\circ}$ so as to ensure identical experimental conditions for both polarizations. From these measurements the angular distributions of both the polarization independent component $I_{0}(\theta)$ and the polarization dependent component $P I_{1}(\theta)$ of the photodisintegration cross section have been deduced and the angular distribution of the asymmetry factor $\Sigma(\theta)$ obtained.

The experimental setup and our data acquisition system are described in Sec. II. In Sec. III a brief review of the pertinent theory is presented and its experimental relevance discussed. Section IV contains a summary of our experimental results, a critical analysis of these results with respect to current theories, and a comparison with earlier experiments. Finally, our main conclusions are summarized in Sec. V.

\section{EXPERIMENTAL SETUP}

There are essentially two independent experimental setups involved in this work: the LADON gamma source on the Adone ring and the actual detection system in an experimental area $42 \mathrm{~m}$ away. The LADON facility will be briefly described first.

\section{A. The gamma beam}

The basic technical features of the LADON system are shown schematically in Fig. 1 (see Refs. 26 and 27 for further details). Under normal laser operation, the harmonic modes-resonating at frequencies $f_{n}=n c / 2 L$ ( $n$ integer) 
TABLE II. $\left[I_{0}\left(\theta_{\mathrm{n}}\right) / \sigma_{t}\right] 10^{2}$ as a function of the neutron c.m. angle $\theta_{\mathrm{n}}=(\pi-\theta)$ and of the laboratory gamma-ray energy $E_{\gamma}(\mathrm{MeV})$.

\begin{tabular}{|c|c|c|c|c|}
\hline$\theta_{\theta_{\mathrm{n}}(\mathrm{deg})} E_{\gamma}(\mathrm{MeV})$ & 19.8 & 29.0 & 38.6 & 60.8 \\
\hline 14.7 & $1.71 \pm 0.26$ & $1.96 \pm 0.11$ & $2.42 \pm 0.21$ & \\
\hline 29.7 & $2.81 \pm 0.22$ & $2.96 \pm 0.20$ & $3.99 \pm 0.34$ & $5.21 \pm 0.38$ \\
\hline 44.5 & $5.79 \pm 0.55$ & $5.03 \pm 0.22$ & $5.04 \pm 0.29$ & \\
\hline 59.3 & $8.36 \pm 0.42$ & $7.82 \pm 0.34$ & $8.44 \pm 0.78$ & $6.36 \pm 0.41$ \\
\hline 89.3 & $11.72 \pm 0.44$ & $10.92 \pm 0.23$ & $10.99 \pm 0.39$ & $9.99 \pm 0.48$ \\
\hline 119.3 & $9.16 \pm 0.44$ & $10.32 \pm 0.41$ & $8.73 \pm 0.50$ & $10.22 \pm 0.50$ \\
\hline 134.5 & $6.91 \pm 0.69$ & $7.57 \pm 0.25$ & $8.08 \pm 0.52$ & \\
\hline 147.6 & $4.08 \pm 0.30$ & $4.96 \pm 0.29$ & $6.03 \pm 0.44$ & $6.22 \pm 0.36$ \\
\hline 163.1 & $2.15 \pm 0.30$ & $2.82 \pm 0.15$ & $3.75 \pm 0.29$ & \\
\hline
\end{tabular}

in an optical cavity of length $L$ that comprises the laser tube-have random phases and the laser power distribution is uniform over the entire cavity. Because the electron-photon scattering takes place only during the passage of an electron bunch through the $5.7 \mathrm{~m}$ long Adone straight section (see Fig. 1), this uniformly distributed laser power remains unused for about $100 \mathrm{nsec}$ of each 117 nsec. The effective laser power can be increased by "compacting" the uniformly distributed laser power into a traveling pulse of phase-locked harmonics. ${ }^{31}$ If the length of the cavity is so adjusted that this laser power packet finds itself going to the right just as an electron bunch crosses it going in the opposite direction (see Fig. 1), the yield of back-scattered photons will increase. ${ }^{27}$

Moreover, in this way we define the laser-electron interaction region in the center of the straight section, where there are no quadrupoles or other ring optical components. In this magnetic-field-free region, the angular divergence of the stored electron beam is minimal, yielding the best monochromaticity for the scattered gamma ray beam.

The cavity mode locking is effected using the piezoelectric property of a quartz crystal for acousto-optic modulation of cavity losses at a frequency $f=c / 2 L$, which is equal to the difference in frequency, $f_{n+1}-f_{n}$, between any two neighboring harmonics. ${ }^{32}$ While the losses associated with the mode-locking process lower the average internal power of the laser cavity by about a factor of 2 (from 60 to about $35 \mathrm{~W}$ in the Coherent Radiation Model
CR-18 Argon-Ion laser we use), the effective laser power overlapping the electron bunch in the electron-photon interaction region is increased by about a factor of 6 . It is this spatial and temporal superposition of the electron bunches and the "compacted" laser power which has raised the LADON beam intensity to the level of $\gtrsim 10^{5}$ photons/sec and which thus made it possible for us to take advantage of its unique properties of tunability and polarization for photonuclear research, particularly for polarization asymmetry measurements.

Other salient features of the LADON gamma source are the following:

(a) its ready tunability in the energy range from $E_{\gamma}=5$ to $78.7 \mathrm{MeV}$, effected by varying the energy of the electrons circulating in the storage ring from $370 \mathrm{MeV}$ to 1.5 $\mathrm{GeV}$;

(b) its nearly perfect polarization (as an example, at $E_{\gamma}=15 \mathrm{MeV}$ the degree of linear polarization is $P=0.997$ theoretically and $P=0.99 \pm 0.02$ as actually measured $^{26}$ ), and a straightforward way to change the plane of polarization to any desired angle relative to the reaction plane;

(c) a very low background of unwanted photons, due essentially only to electron bremsstrahlung off the residual gas molecules in the $10^{-9}$ Torr vacuum of the Adone ring.

The energy resolution varies from $\sim 2 \%$ FWHM at lower gamma energies, increasing up to $8 \%$ at $E_{\gamma}=70$ $\mathrm{MeV}$. The reasons for the decrease of resolution with in-

TABLE III. $\left[I_{1}\left(\theta_{\mathrm{n}}\right) / \sigma_{t}\right] 10^{2}$ as a function of the neutron c.m. angle $\theta_{\mathrm{n}}=(\pi-\theta)$ and of the laboratory gamma-ray energy $E_{\gamma}(\mathrm{MeV})$.

\begin{tabular}{ccccc}
\hline \hline$\theta_{\mathrm{n}}(\mathrm{deg})$ & 19.8 & 29.0 & 38.6 & 60.8 \\
\hline 14.7 & & & & \\
29.7 & $0.63 \pm 0.22$ & $0.62 \pm 0.12$ & $0.32 \pm 0.26$ & \\
44.5 & $2.11 \pm 0.21$ & $1.66 \pm 0.21$ & $1.81 \pm 0.35$ & $0.80 \pm 0.37$ \\
59.3 & $4.72 \pm 0.51$ & $3.65 \pm 0.23$ & $2.54 \pm 0.29$ & \\
89.3 & $7.11 \pm 0.41$ & $6.06 \pm 0.35$ & $5.62 \pm 0.79$ & $2.07 \pm 0.40$ \\
119.3 & $10.74 \pm 0.44$ & $8.94 \pm 0.23$ & $7.74 \pm 0.40$ & $4.97 \pm 0.46$ \\
134.5 & $8.01 \pm 0.44$ & $8.31 \pm 0.41$ & $6.29 \pm 0.50$ & $5.08 \pm 0.49$ \\
147.6 & $5.66 \pm 0.62$ & $5.57 \pm 0.27$ & $5.05 \pm 0.53$ & \\
163.1 & $3.00 \pm 0.29$ & $3.05 \pm 0.29$ & $3.40 \pm 0.44$ & $1.56 \pm 0.39$ \\
\hline \hline
\end{tabular}


TABLE IV. The azimuthal asymmetry factor $\Sigma\left(\theta_{\mathrm{n}}\right)=I_{1}\left(\theta_{\mathrm{n}}\right) / I_{0}\left(\theta_{\mathrm{n}}\right)$ as a function of the neutron c.m. angle $\theta_{\mathrm{n}}=(\pi-\theta)$ and of the laboratory gamma-ray energy $E_{\gamma}(\mathrm{MeV})$.

\begin{tabular}{|c|c|c|c|c|}
\hline$\overbrace{\theta_{\mathrm{n}}(\mathrm{deg})} E_{\gamma}(\mathrm{MeV})$ & 19.8 & 29.0 & 38.6 & 60.8 \\
\hline 14.7 & $0.370 \pm 0.054$ & $0.316 \pm 0.053$ & $0.134 \pm 0.104$ & \\
\hline 29.7 & $0.752 \pm 0.036$ & $0.562 \pm 0.051$ & $0.453 \pm 0.076$ & $0.154 \pm 0.064$ \\
\hline 44.5 & $0.816 \pm 0.020$ & $0.725 \pm 0.023$ & $0.504 \pm 0.050$ & \\
\hline 59.3 & $0.851 \pm 0.022$ & $0.775 \pm 0.024$ & $0.666 \pm 0.072$ & $0.326 \pm 0.053$ \\
\hline 89.3 & $0.917 \pm 0.007$ & $0.819 \pm 0.013$ & $0.704 \pm 0.020$ & $0.497 \pm 0.039$ \\
\hline 119.3 & $0.874 \pm 0.019$ & $0.805 \pm 0.021$ & $0.721 \pm 0.036$ & $0.497 \pm 0.043$ \\
\hline 134.5 & $0.819 \pm 0.019$ & $0.736 \pm 0.018$ & $0.625 \pm 0.050$ & \\
\hline 147.6 & $0.737 \pm 0.030$ & $0.615 \pm 0.041$ & $0.564 \pm 0.057$ & $0.251 \pm 0.067$ \\
\hline 163.1 & $0.482 \pm 0.062$ & $0.320 \pm 0.046$ & $0.344 \pm 0.076$ & \\
\hline
\end{tabular}

creasing energy are twofold: the relativistic squeezing of a wider energy range of scattered photons into the forward solid angle of $\Omega=2.5 \times 10^{-8} \mathrm{sr}$ defined by the beam collimation system, and the increasing divergence of the electron beam. These two effects set a practical upper limit to the "monochromaticity" of gamma beams produced by Compton back scattering. In Table I the $\gamma$ beam characteristics at the energies of our experiment are reported.
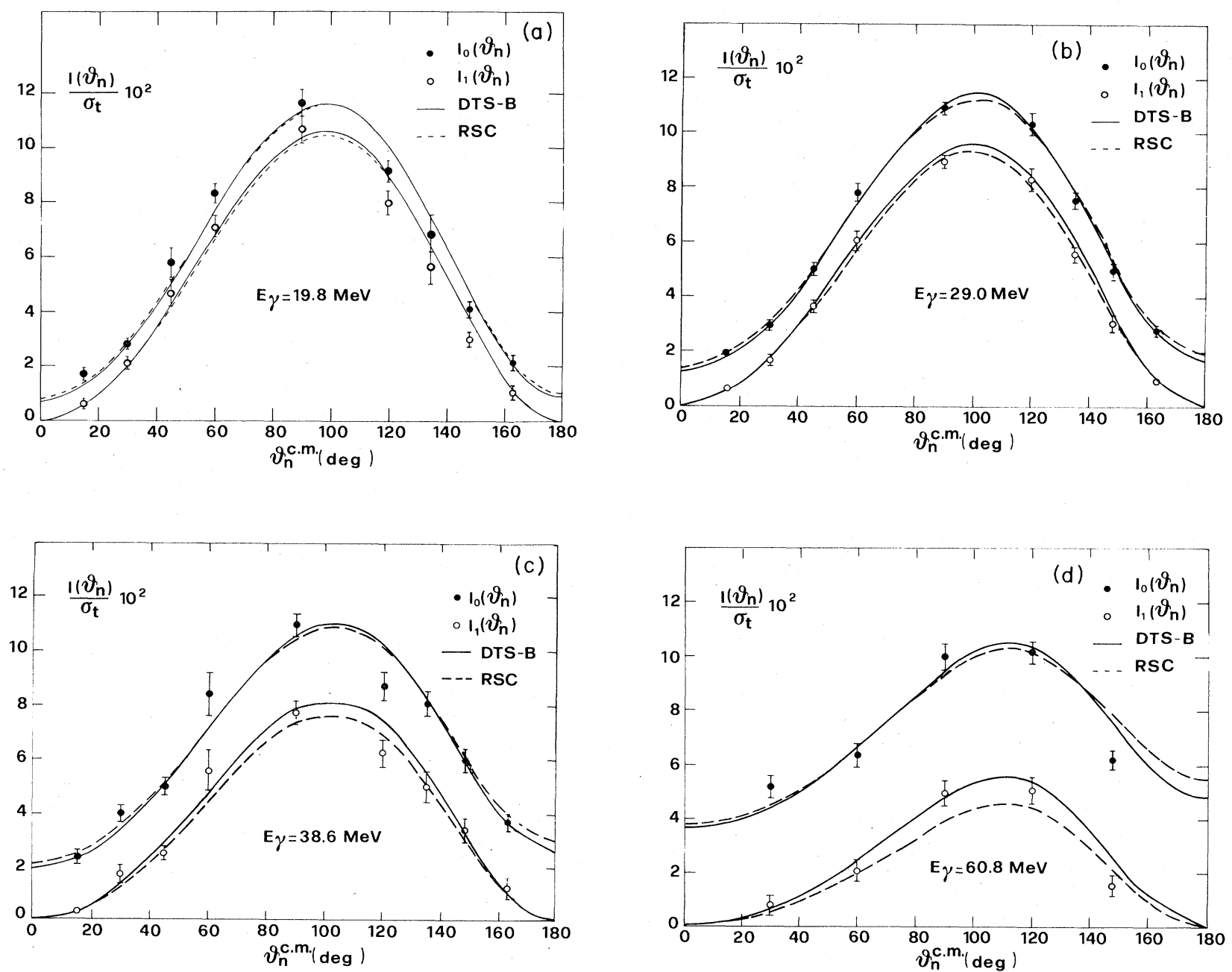

FIG. 4. Plots of $\left[I_{0}\left(\theta_{\mathrm{n}}\right) / \sigma_{t}\right] 10^{2}$ and of $\left[I_{1}\left(\theta_{\mathrm{n}}\right) / \sigma_{t}\right] 10^{2}$ as a function of the c.m. neutron angles $\theta_{\mathrm{n}}=(\pi-\theta)$ at $E_{\gamma}=(\mathrm{a}) 19.8 \mathrm{MeV}$; (b) $29.0 \mathrm{MeV}$; (c) $38.6 \mathrm{MeV}$; (d) $60.8 \mathrm{MeV}$. Dashed and solid lines represent theoretical calculations of Refs. 10 and 11 with the RSC and the DTS-B potentials, respectively. 

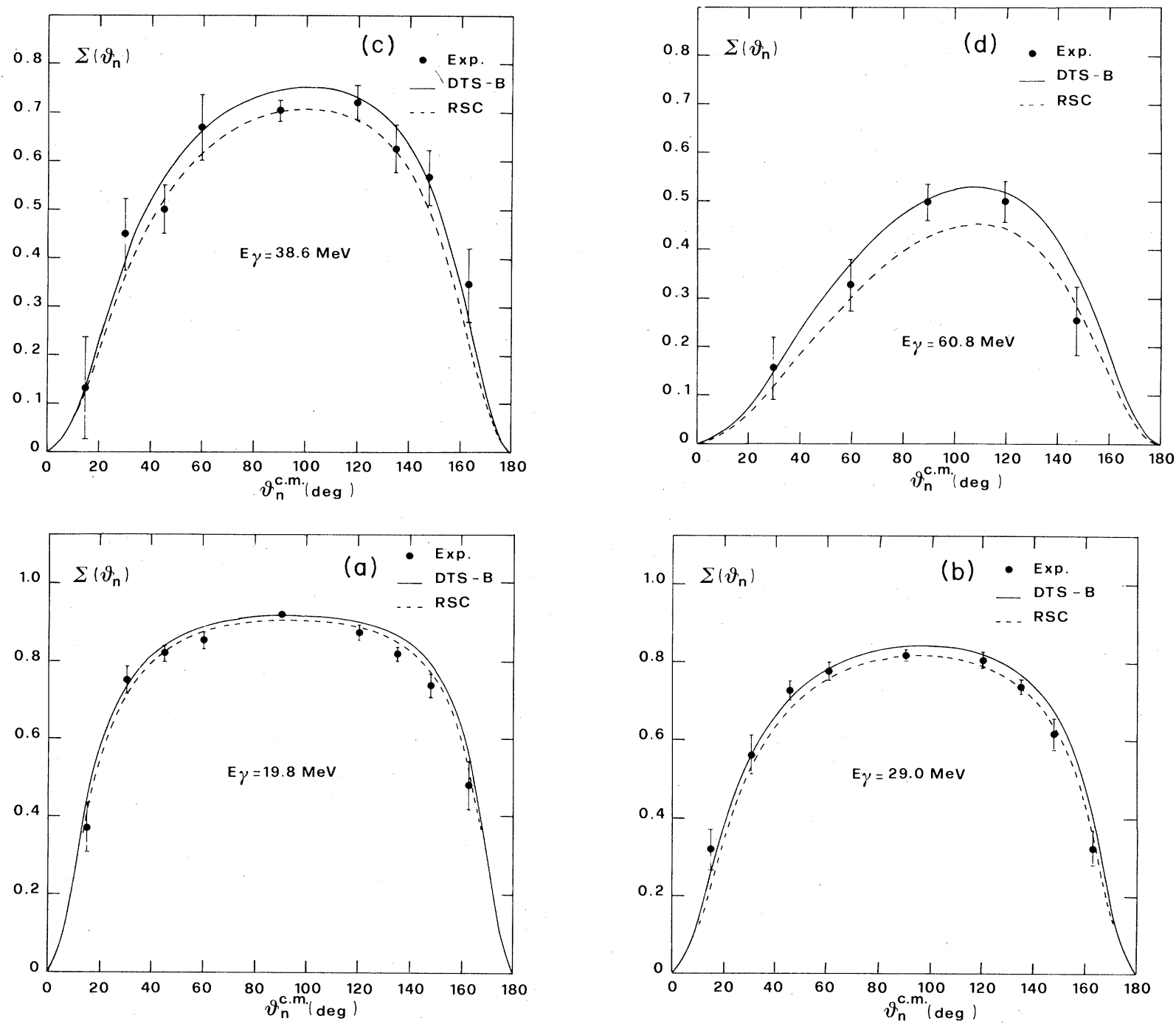

FIG. 5. Plots of the azimuthal asymmetry factor $\Sigma\left(\theta_{\mathrm{n}}\right)$ as a function of the c.m. neutron angles $\theta_{\mathrm{n}}=(\pi-\theta)$. The meaning of symbols is the same as in Fig. 4.

\section{B. Experiment}

The layout of the experimental area is shown in Fig. 2. After leaving the interaction region, the gamma beam passes a system of collimators which limits it to the forward solid angle of $2.5 \times 10^{-8} \mathrm{sr}$, and a set of three sweeping magnets that clean the beam of charged parti- cles. During the entire experiment, the beam energy spectrum is analyzed by a magnetic pair spectrometer, the output of which is evaluated on-line by a microprocessor. The target consists of a $C_{6} D_{6}$ deuterated liquid scintillator NE-230 in a cylindrical container $3.81 \mathrm{~cm}$ in diameter and $10.16 \mathrm{~cm}$ in height, which serves at the same time as proton detector, optically coupled to a 56-AVP photomulti-

TABLE V. Coefficients of the expansion for $\left[I_{0}(\theta) / \sigma_{t}\right] 10^{2}$ given by Eqs. $(4 \mathrm{a})$ and $(5 \mathrm{a})$.

\begin{tabular}{lcrcc}
\hline \hline$E_{\gamma}(\mathrm{MeV})$ & 19.8 & 29.0 & 38.6 & 60.8 \\
\hline$\left(A_{1} / \sigma_{t}\right) 10^{2}$ & $0.58 \pm 0.15$ & $1.34 \pm 0.18$ & $1.18 \pm 0.42$ & $1.62 \pm 0.7$ \\
$\left(A_{2} / \sigma_{t}\right) 10^{2}$ & $-7.05 \pm 0.34$ & $-6.32 \pm 0.19$ & $-5.95 \pm 0.51$ & $-3.7 \pm 1.0$ \\
$\left(A_{3} / \sigma_{t}\right) 10^{2}$ & $-0.48 \pm 0.16$ & $-0.76 \pm 0.17$ & $-1.1 \pm 0.55$ & $-0.8 \pm 0.9$ \\
$\left(A_{4} / \sigma_{t}\right) 10^{2}$ & $-0.14 \pm 0.15$ & $-0.07 \pm 0.19$ & $-0.44 \pm 0.18$ & $-0.37 \pm 0.2$ \\
& & & & \\
$\left(a / \sigma_{t}\right) 10^{2}$ & $0.8 \pm 0.1$ & $1.59 \pm 0.08$ & $1.75 \pm 0.15$ & $3.70 \pm 0.55$ \\
$\left(b / \sigma_{t}\right) 10^{2}$ & $11.47 \pm 0.15$ & $9.74 \pm 0.24$ & $10.60 \pm 0.52$ & $7.79 \pm 0.85$ \\
$\left(c / \sigma_{t}\right) 10^{2}$ & $0.09 \pm 0.08$ & $0.186 \pm 0.14$ & $0.247 \pm 0.2$ & 0.608 \\
$\left(d / \sigma_{t}\right) 10^{2}$ & $1.26 \pm 0.5$ & $2.77 \pm 0.51$ & $2.55 \pm 0.75$ & $1.99 \pm 1.0$ \\
$\left(e / \sigma_{t}\right) 10^{2}$ & $-0.888 \pm 0.7$ & $-0.22 \pm 0.56$ & $-1.62 \pm 0.7$ & $-1.75 \pm 0.65$ \\
\hline \hline
\end{tabular}




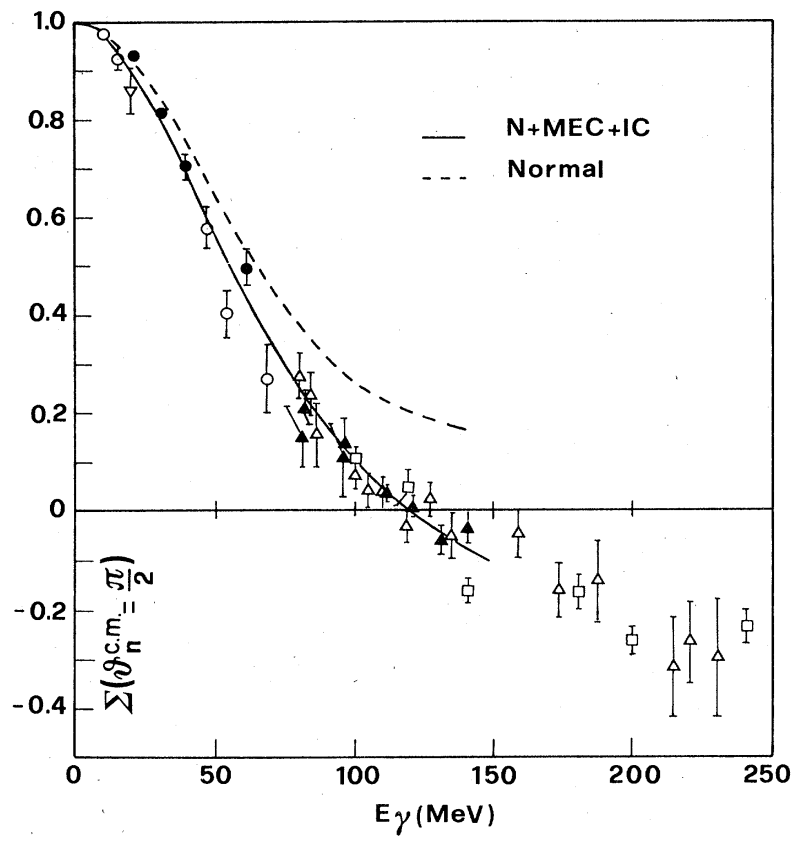

FIG. 6. Plot of the asymmetry factor $\Sigma\left(\theta_{n}=\pi / 2\right)$ vs laboratory gamma-ray energy (MeV). Our present data (solid circles) are compared with the results of earlier experiments of Liu (solid and open triangles, Ref. 39), Del Bianco et al. (inverted open triangle, Ref. 41), our previous data (open circles, Ref. 43), and Gorbenko et al. (open squares, Ref. 44). The theoretical curves have been obtained in Ref. 10 with the RSC potential. The dashed line corresponds to the standard Partovi approximation (Ref. 49); the solid line reflects the inclusion of MEC and IC corrections.

plier.

Five neutron detectors, independently movable in the reaction plane, cover an angular range from $\sim 15$ to $\sim 165$ deg. Each neutron detector consists of a cylindrical container $30 \mathrm{~cm}$ in diameter and $15 \mathrm{~cm}$ deep filled with an NE-213 liquid scintillator $\left(\mathrm{C}_{6} \mathrm{H}_{6}\right)$ and each coupled to a 58-DVP photomultiplier. The beam intensity is continuously monitored by a lead glass counter which also serves as gamma beam stopper.

The following data are collected via Camac and recorded by a PDP $11 / 34$ for each event:

(i) The "head" integral $H$ of each pulse from the proton counter (obtained by integrating the entire pulse) and the corresponding "tail" integral $T$ (obtained by starting a second integration $25 \mathrm{nsec}$ after the pulse start). These data, processed subsequently off line, permit us to suppress most of the considerable gamma background of the proton detectors by effective pulse-shape discrimination.

(ii) Similar "head" and "tail" integrals are recorded for each pulse from any of the five neutron counters, together with the corresponding time of flight and the code number of the particular counter. The time of flight records constitute the essential part of our experimental data.

The information from the proton counter and from the neutron counters is stored only if two coincidence conditions are satisfied:

(i) A fast coincidence ( $\sim 20$ nsec window) between the electron beam pulse and a signal from the proton counter.

(ii) A slower coincidence ( $\sim 80$ nsec window) between the output of the fast coincidence and any of the neutron counters.

The most important contributions to the background are due to electromagnetic processes (multiple Compton scattering, pair production, and annihilation) involving atomic electrons. These processes give mainly rise to narrow peaks in the time of flight spectra of the neutron counters: By using a TPHC-SCA (time to pulse height conversion single channel analyzer) to clear the Camac modules whenever the time of flight is in the "gammalike" range, the sharp peaks are suppressed.

The first step in the off-line data processing involves reconstruction of the "raw" neutron time of flight spectra for each of the five neutron detectors. For each energy and each neutron angle there are two sets of such spectra, one for $\varphi=0^{\circ}$ and one for $\varphi=90^{\circ}$, each obtained by adding the results of a number of corresponding runs. Each "raw" time of flight spectrum includes a residual gamma background, having a flat time structure which is effectively suppressed by pulse shape discrimination. Still further background reduction is effected by imposing a pulse height window onto the signals from the proton ADC (analog to digital converter) and by rejecting signals below a fixed threshold from the neutron ADC. A typical result is shown in Fig. 3. A residual background remains, mostly due to the extreme tails of the gamma peak, the carbon in the target, bremsstrahlung events, and cosmic rays.

\section{THEORETICAL CONSIDERATIONS}

The general expression of the differential cross section for the ${ }^{2} \mathbf{H}(\gamma, p)$ n process is given in perturbation theory by

$$
d \sigma / d \Omega=\left(E_{\gamma} M / 8 \pi^{2}\right)\left|\left\langle\psi_{f}\left|H_{\text {int }}\right| \psi_{i}\right\rangle\right|^{2},
$$

TABLE VI. Coefficients of the expansion for $\left[I_{1}(\theta) / \sigma_{t}\right] 10^{2}$ given by Eqs. (4b) and (5b).

\begin{tabular}{lcccc}
\hline \hline$E_{\gamma}(\mathrm{MeV})$ & 19.8 & \multicolumn{1}{c}{29.0} & 38.6 & 60.8 \\
\hline$\left(B_{2} / \sigma_{t}\right) 10^{2}$ & $3.47 \pm 0.05$ & $3.05 \pm 0.06$ & $2.57 \pm 0.16$ & $1.61 \pm 0.2$ \\
$\left(B_{3} / \sigma_{t}\right) 10^{2}$ & $0.075 \pm 0.02$ & $0.156 \pm 0.02$ & $0.188 \pm 0.03$ & $0.187 \pm 0.04$ \\
$\left(B_{4} / \sigma_{t}\right) 10^{2}$ & $-(1.24 \pm 0.5) 10^{-2}$ & $(0.732 \pm 0.55) 10^{-2}$ & $(1.25 \pm 1.2) 10^{-2}$ & $-(0.8 \pm 1.8) 10^{-2}$ \\
& & & & \\
$\left(f / \sigma_{t}\right) 10^{2}$ & $10.13 \pm 0.12$ & $9.51 \pm 0.16$ & $8.38 \pm 0.58$ & $4.51 \pm 0.57$ \\
$\left(g / \sigma_{t}\right) 10^{2}$ & $1.16 \pm 0.33$ & $2.37 \pm 0.34$ & $2.89 \pm 0.33$ & $2.84 \pm 0.46$ \\
$\left(h / \sigma_{t}\right) 10^{2}$ & $0.297 \pm 0.2$ & $-0.397 \pm 0.25$ & $-0.79 \pm 0.75$ & $0.371 \pm 1.0$ \\
\hline \hline
\end{tabular}



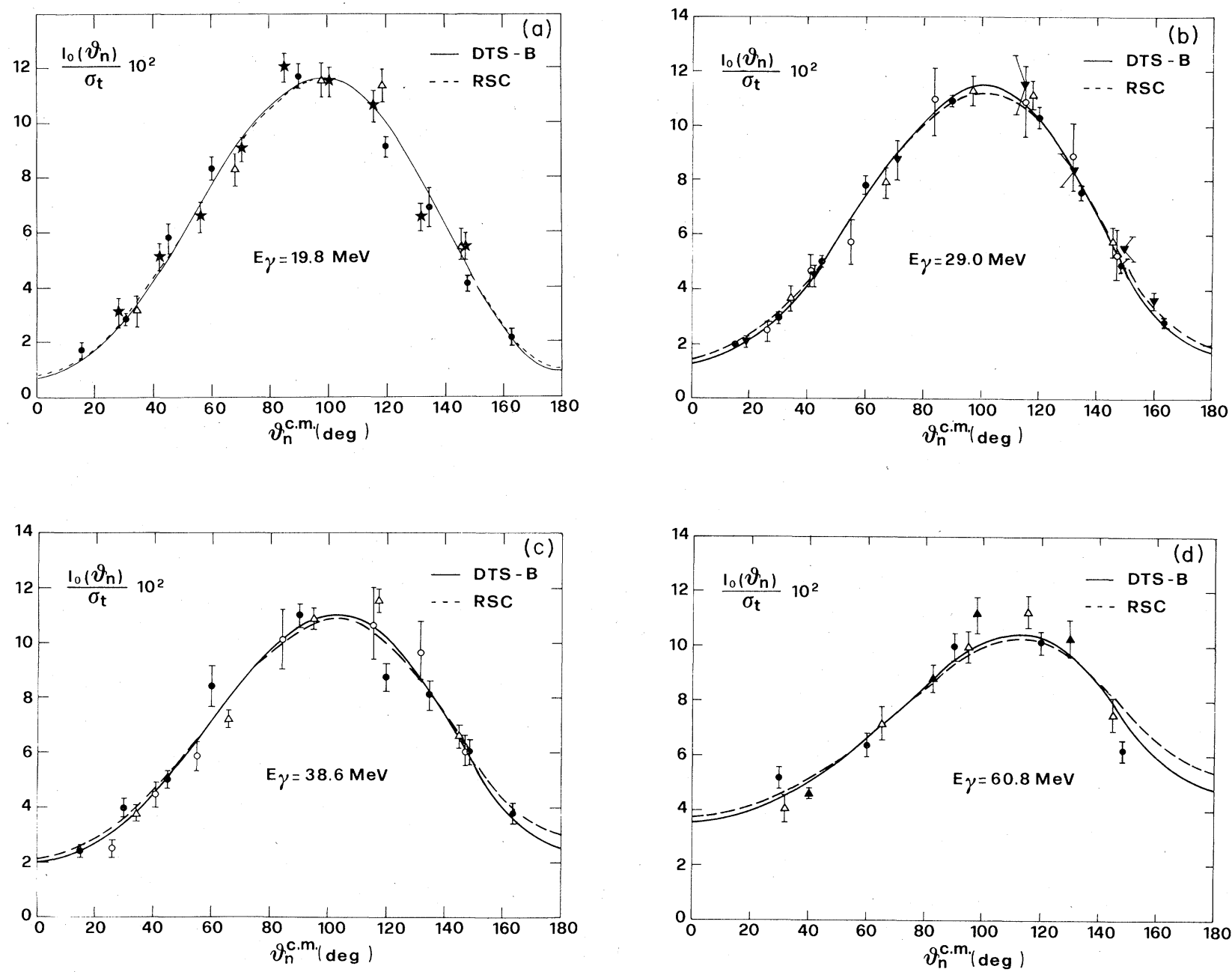

FIG. 7. A survey of the experimental determinations of $\left[I_{0}\left(\theta_{\mathrm{n}}\right) / \sigma_{t}\right] 10^{2}$ for $E_{\gamma}=(\mathrm{a}) 19.8 \mathrm{MeV}$; (b) $29.0 \mathrm{MeV}$; (c) $38.6 \mathrm{MeV}$; (d) 60.8 $\mathrm{MeV}$. Our data (solid circles) are compared with results of earlier measurements of Halpern and Weinstock (stars, Ref. 50); Shin et al. (open triangles, Ref. 51); Weissman et al. (open circles, Ref. 33); Allen (inverted solid triangles, Ref. 52); and Galey (solid triangles, Ref. 53). Corresponding theoretical curves obtained in Refs. 10 and 11 with the RSC (dashed lines) and the DTS-B (solid lines) potentials are also shown.

where $\psi_{i}$ is the nonrelativistic deuteron wave function and $\psi_{f}$ represents the n-p final state with its interaction. For linearly polarized photons, the above expression is usually reduced, ${ }^{11}$ in the center of mass (c.m.) system, to the form

$$
\begin{aligned}
d \sigma / d \Omega & =I_{0}(\theta)+P I_{1}(\theta) \cos 2 \varphi \\
& =I_{0}(\theta)[1+P \Sigma(\theta) \cos 2 \varphi],
\end{aligned}
$$

where $\varphi$ is the angle between the ${ }^{2} \mathrm{H}(\gamma, \mathrm{p}) \mathrm{n}$ reaction and polarization planes and $\theta$ is the c.m. angle between the proton and photon momenta (if neutrons rather than protons are detected, $\theta=\theta_{\mathrm{p}}$ must be replaced by $\theta_{\mathrm{n}}=\pi-\theta$, but otherwise the expression remains unchanged), $P$ represents the degree of linear polarization of the photon beam, and

$$
\Sigma(\theta)=I_{1}(\theta) / I_{0}(\theta)
$$

is a parameter which describes the azimuthal asymmetry in the differential cross section due to the polarization of the incident beam. $\Sigma(\theta)$ has been shown ${ }^{10}$ to be markedly affected by mesonic degrees of freedom. It should be noted that $I_{1}(\theta)$ and $\Sigma(\theta)$ are accessible to experimental determination only with polarized $\gamma$ beams.

The quantities $I_{0}(\theta)$ and $I_{1}(\theta)$ of Eq. (2) may be expanded in terms of Legendre polynomials $P_{J}(\cos \theta)$ and associated Legendre functions $P_{J}^{m}(\cos \theta),{ }^{11}$ respectively. If one includes multipole contributions up to the dipoleoctupole interference, one has

$$
\begin{aligned}
& I_{0}(\theta)=\sum_{i=0}^{4} A_{i}\left(E_{\gamma}\right) P_{i}(\cos , \theta), \\
& I_{1}(\theta)=\sum_{i=2}^{4} B_{i}\left(E_{\gamma}\right) P_{i}^{2}(\cos , \theta),
\end{aligned}
$$

where $A_{i}\left(E_{\gamma}\right)$ and $B_{i}\left(E_{\gamma}\right)$ are energy dependent coefficients.

An alternative way in which the functions $I_{0}(\theta)$ and $I_{1}(\theta)$ may be written ${ }^{11,49}$ is 

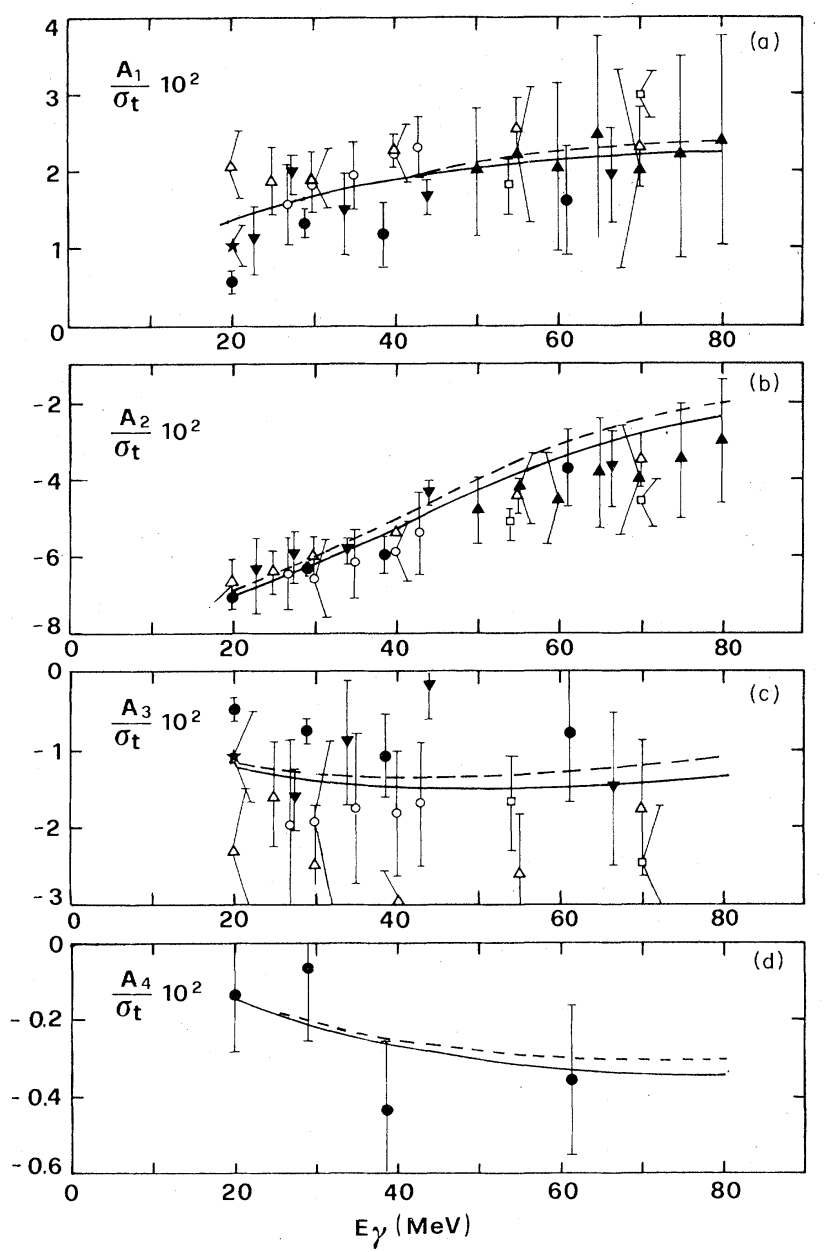

FIG. 8. Plots of a representative sampling of coefficients $A_{i} / \sigma_{t}$ [see Eq. (4a)] vs the laboratory gamma-ray energy $(\mathrm{MeV})$. Our data (solid circles) are compared with results of measurements by other groups; all symbols have the same meaning as in Fig. 7. In addition, the open squares represent the data of Alexandrov et al., Ref. 56. Corresponding theoretical curves obtained in Refs. 10 and 11 with the RSC (dashed lines) and the DTS-B (solid lines) potentials are also shown.

$$
I_{0}(\theta)=a+b \sin ^{2} \theta+c \cos \theta+d \sin ^{2} \theta \cos \theta+e \sin ^{4} \theta,
$$$$
I_{1}(\theta)=f \sin ^{2} \theta+g \sin ^{2} \theta \cos \theta+h \sin ^{4} \theta,
$$

where the energy dependent coefficients are now $a, b, c, \ldots, h$. The relationship between these and the previous coefficients is given by ${ }^{11}$

$$
\begin{aligned}
& a=A_{0}+A_{2}+A_{4}, \quad b=-\left(3 A_{2}+10 A_{4}\right) / 2, \\
& c=A_{1}+A_{3}, \quad d=-5 A_{3} / 2, \\
& e=35 A_{4} / 8, \quad f=3\left(B_{2}+15 B_{4}\right), \\
& g=15 B_{3}, \quad h=-105 B_{4} / 2 .
\end{aligned}
$$

While the two forms of expressions for $I_{0}(\theta)$ and $I_{1}(\theta)$ are equivalent, the coefficients $A_{i}$ and $B_{i}$ of Eqs. (4) are
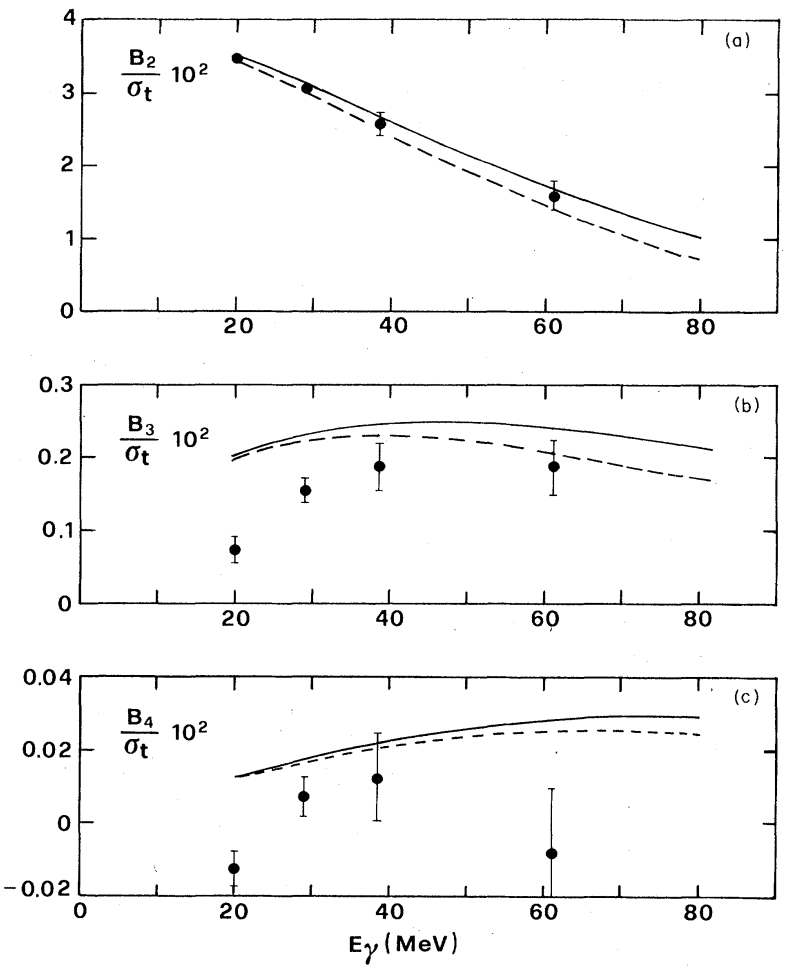

FIG. 9. Plots of the coefficients $B_{i} / \sigma_{t}$ [see Eq. (4b)] vs the laboratory gamma-ray energy $(\mathrm{MeV})$. Our data for $B_{2} / \sigma_{t}$, $B_{3} / \sigma_{t}$, and $B_{4} / \sigma_{t}$ (solid circles) are compared with results of theoretical calculations of Refs. 10 and 11 based on the RSC (dashed lines) and DTS-B (solid lines) potentials, respectively.

better suited for comparison between theory and experiment because of the orthogonality of the Legendre functions. ${ }^{34}$ Still, the expressions of Eqs. (5) can be useful for extracting additional information regarding nuclear potentials, as will be seen in Sec. IV.

In the present experiment, angular distributions in $\theta_{\mathrm{n}}$ of neutrons were taken and measurements were made for two polarization angles, $\varphi=0$ and $\varphi=\pi / 2$. Under these conditions, Eq. (2) reduces to

$$
d \sigma / d \Omega=I_{0}\left(\theta_{\mathrm{n}}\right)+P I_{1}\left(\theta_{\mathrm{n}}\right)
$$

for $\varphi=0$, and to

$$
d \sigma / d \Omega=I_{0}\left(\theta_{\mathrm{n}}\right)-P I_{1}\left(\theta_{\mathrm{n}}\right)
$$

for $\varphi=\pi / 2$.

Thus the values of $I_{0}\left(\theta_{\mathrm{n}}\right)$ and $I_{1}\left(\theta_{\mathrm{n}}\right)$ may be obtained from the experimental data by the following relations:

$I_{0}\left(\theta_{\mathrm{n}}\right)=\frac{1}{2} \alpha\left[K_{1} Y\left(\theta_{\mathrm{n}}, \varphi=0\right)+K_{2} Y\left(\theta_{\mathrm{n}}, \varphi=\pi / 2\right)\right]$,

$I_{1}\left(\theta_{\mathrm{n}}\right)=\frac{1}{2} \alpha\left[K_{1} Y\left(\theta_{\mathrm{n}}, \varphi=0\right)-K_{2} Y\left(\theta_{\mathrm{n}}, \varphi=\pi / 2\right)\right]$,

where $Y\left(\theta_{n}, \varphi\right)$ is the experimental neutron yield (the ratio between the number of photoneutrons detected at $\theta_{\mathrm{n}}$ and the gamma-ray flux). The corrective coefficients $K_{1}$ and $K_{2}$, evaluated by Monte Carlo methods, account for neutron absorption, multiple scattering, and finite solid angle. In the present work the $\alpha$ constant has been fixed to nor- 

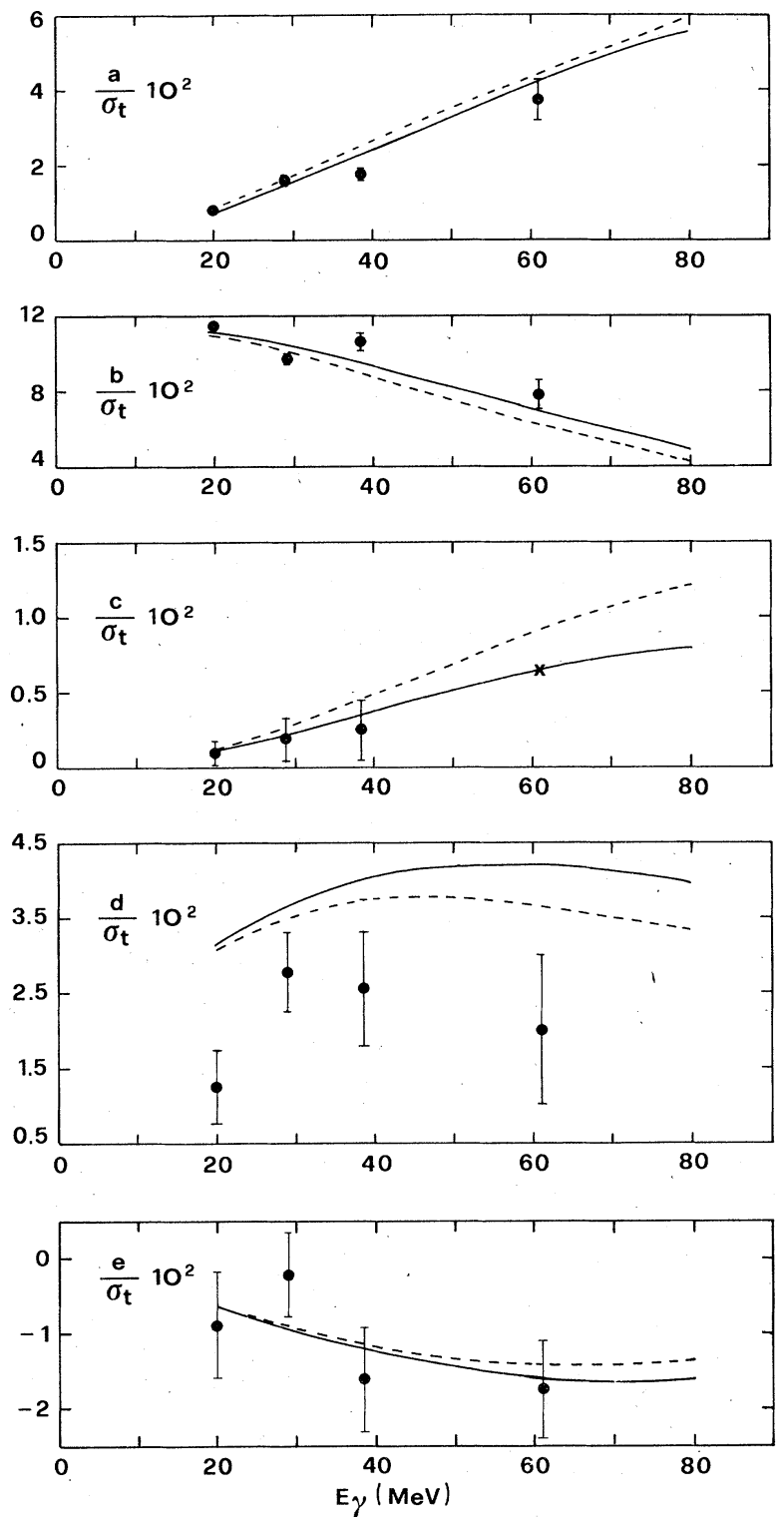

FIG. 10. Plots of the coefficients $a / \sigma_{t}, b / \sigma_{t}, c / \sigma_{t}, d / \sigma_{t}$, and $e / \sigma_{t}$ [see Eq. (5a)] vs the laboratory gamma-ray energy $(\mathrm{MeV})$. Our data (solid circles) are compared with the theoretical curves obtained in Refs. 10 and 11 with the RSC (dashed lines) and the DTS-B (solid lines) potentials, respectively.

malize our data in such a way that $\int I_{0}\left(\theta_{\mathrm{n}}\right) d \Omega_{\mathrm{n}}=1$; consequently, both $I_{0}\left(\theta_{n}\right)$ and $I_{1}\left(\theta_{n}\right)$ are given in units of the total cross section for unpolarized photons $\left[\sigma_{t}=\int I_{0}\left(\theta_{\mathrm{n}}\right) d \Omega_{\mathrm{n}}\right]$, without being affected by any uncertainty both in the neutron counter efficiency and in the photon beam monitoring.

By defining

$$
R\left(\theta_{\mathrm{n}}\right)=Y\left(\theta_{\mathrm{n}}, \pi / 2\right) / Y\left(\theta_{\mathrm{n}}, 0\right)
$$

and writing $K=K_{2} / K_{1}$, we obtain a form of Eq. (3) which is directly linked with the neutron yields obtained in the experiment:
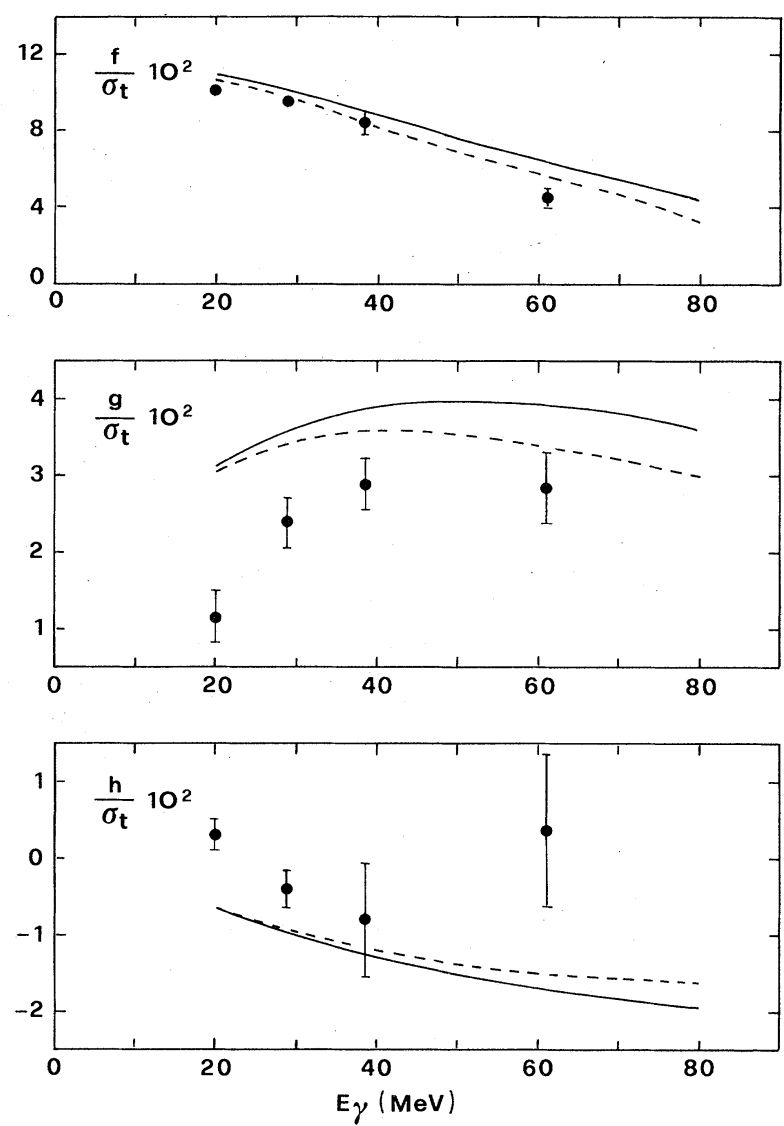

FIG. 11. Plots of the coefficients $f / \sigma_{t}, g / \sigma_{t}$, and $h / \sigma_{t}$ [see Eq. (5b)] vs the laboratory gamma-ray energy (MeV). Our data for $f / \sigma_{t}, g / \sigma_{t}$, and $h / \sigma_{t}$ are compared with theoretical curves obtained in Refs. 10 and 11 with the RSC (dashed lines) and the DTS-B (solid lines) potentials, respectively.

$$
\Sigma\left(\theta_{\mathrm{n}}\right)=\frac{1-K R\left(\theta_{\mathrm{n}}\right)}{P\left[1+K R\left(\theta_{\mathrm{n}}\right)\right]} .
$$

As previously pointed out, all available calculations clearly indicate that, to account accurately for the experi-

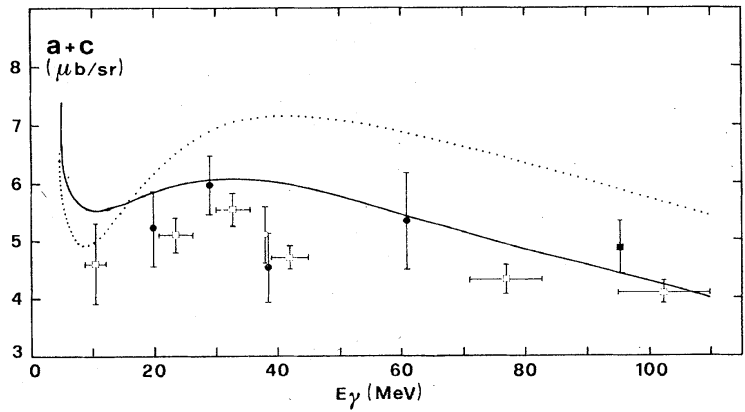

FIG. 12. Plot of our estimates (full circles), for the forward cross section $(a+c)$, compared with the experimental results of Refs. 36 and 57 (open squares), Refs. 37 and 58 (open circle), and Ref. 38 (full square). The theoretical calculations are due to Cambi et al. (Ref. 11) in IA (dotted line) and with relativistic corrections (full line) using the Paris potential. 
mental data at photon energies above some 30 or $40 \mathrm{MeV}$, meson exchange currents (MEC) and nucleon isobar contributions (IC) must be taken into account in the evaluation of the expansion coefficients. The functions $I_{1}(\theta)$ and $\Sigma(\theta)$ are particularly sensitive to the MEC and IC corrections. For this reason, the ${ }^{2} \mathbf{H}(\gamma, \mathrm{p}) \mathbf{n}$ reaction with polarized gammas turns out to be a sensitive probe for checking the standard perturbative calculations of the MEC and IC effects.

Another important aspect of ${ }^{2} \mathbf{H}(\gamma, p)$ n studies involves the forward and backward differential cross section for unpolarized photons, respectively given by $I_{0}(\theta=0)$ and $I_{0}(\theta=\pi)$. These values are strongly dependent on the percentage of the $P_{D}$ admixture $e^{19,20,23}$ in the deuteron wave function. Consequently experimental determination may offer a way for better defining the medium range shape of the tensor component of the N-N interaction.

The calculations of $I_{0}(\theta)$ are usually carried out under the assumption that the charge density operator may be expressed as a sum of standard one-body terms, and that two-body and relativistic corrections may be neglected (the Siegert hypothesis). Because the values of the forward and backward differential cross sections are highly sensitive to the presence of relativistic and exchange contributions to the nucleon charge density operator, ${ }^{15,21,22,24,25}$ corresponding experimental data may help to ascertain the validity limits of the Siegert hypothesis.

Unfortunately, the above theoretical framework cannot be accurately tested by existing experimental data. In almost all experiments carried out so far, values of $I_{0}(\theta)$ have been measured only in the angular region of $25^{\circ} \leq \theta \leq 150^{\circ}$ (for a complete listing see Ref. 35). The notable exceptions are the measurements of $I_{0}(0)$ and $I_{0}(\pi)$ carried out by Hughes et al., ${ }^{36}$ by Gilot et al., ${ }^{37}$ by Meyer et al., ${ }^{38}$ and by Zieger et al.,${ }^{57}$ and a few measurements of $\Sigma(\theta) .{ }^{39-44}$ Moreover, all the available measurements of $I_{0}(\theta)$, except those carried out at the LADON facility in Frascati, have been produced by nonmonochromatic beams with results that are not always consistent. Moreover, the measurements of $\Sigma(\theta)$ are affected by uncertainties stemming from a poor knowledge of the degree of polarization $P$. Particular problems for the theoretical examination of the ${ }^{2} \mathrm{H}(\gamma, p)$ n reaction derive from the lack of reliable and firm experimental values for $I_{0}(\theta)$ and from the insufficient experimental information on $\Sigma(\theta)$ at energies $E_{\gamma} \lesssim 70 \mathrm{MeV}$. It is the purpose of our research program to remedy this situation, at least partially.

\section{EXPERIMENTAL RESULTS AND DISCUSSION}

Our experimental data are listed as a function of energy and angle in Tables II-IV. All reported results represent the statistically weighted mean of a number (from two to five) of separated measurements. Quoted errors are mainly due to counting statistics; in a few cases (a few extreme $\theta_{\mathrm{n}}$ angles), the errors deriving from uncertainties in the subtraction of the residual flat background in the time of flight spectra had also to be taken into account. Systematic errors essentially derive from the Monte Carlo evaluation of the $K_{1}$ and $K_{2}$ correction factors [see Eqs.
(9) and (10)], as due to the uncertainties in the knowledge of the neutron cross sections and the Monte Carlo statistical accuracies. On the other hand, their ratio $K$, which directly intervenes in Eq. (12), is always very close to unity, and their $\theta$ dependence appearing in Eqs. (9) and (10) is never very strong. Conservative estimates lead us to conclude that, assuming $\Delta K_{1} / K_{1}=\Delta K_{2} / K_{2}$,

$$
\begin{aligned}
& \left(\Delta I_{0}\right)_{\text {syst }} / I_{0} \lesssim \frac{\Delta K_{1}}{K_{1}}\left[\frac{1+\Sigma^{2}}{2}\right]^{1 / 2}, \\
& \left(\Delta I_{1}\right)_{\text {syst }} / I_{1} \sim \frac{\left(\Delta I_{0}\right)_{\text {syst }}}{\Sigma I_{0}}, \\
& (\Delta \Sigma)_{\text {syst }} \sim 2^{-1 / 2} \frac{\Delta K_{1}}{K_{1}}\left(1-\Sigma^{2}\right) .
\end{aligned}
$$

Thus, by assuming $\Delta K_{1} / K_{1} \simeq 5 \%$, systematic errors are expected to be of the same order as the statistical errors around $\theta=\pi / 2$, and considerably lower elsewhere.

Our results for $\left(I_{0} / \sigma_{t}\right) 10^{2}$ and $\left(I_{1} / \sigma_{t}\right) 10^{2}$ as a function of the c.m. neutron angle $\theta_{\mathrm{n}}$, for the four laboratory photon energies used in our experiment, are plotted in Figs. 4(a) -(d). The behavior of the azimuthal asymmetry factor $\Sigma\left(\theta_{n}\right)$ is shown in Figs. 5(a)-(d).

For comparison purposes, two theoretical curves are presented in all figures: the dashed lines have been obtained by Arenhövel, ${ }^{5,10}$ and independently by Cambi et al., ${ }^{11,16}$ using the Reid soft-core (RSC) N-N potential. ${ }^{45}$ According to Ref. 11, no appreciable difference with respect to this result is obtained by using the Hamada-Johnston ${ }^{46}$ or the Paris potential. ${ }^{47}$ On the other hand, the full lines ${ }^{11,16}$ obtained by using the version B of the DeTourreil-Sprung (DTS-B) potential ${ }^{48}$ are higher as far as $I_{1}\left(\theta_{\mathrm{n}}\right)$ and $\Sigma\left(\theta_{\mathrm{n}}\right)$ are concerned, the difference increasing with the energy. In all these calculations, multipoles up to $L=4$ have been included and MEC (and where indicated also IC) contributions have been added to the standard (or "normal") Partovi theory. ${ }^{49}$ These MEC effects are more pronounced in $I_{1}(\theta)$ and $\Sigma(\theta)$ than in $I_{0}(\theta)$ and rapidly increase with $E_{\gamma}$ energy. Relativistic or two-body corrections have not been added to the usual one-body charge operator (the Siegert hypothesis).

The role of the mesonic and isobar degrees of freedom in this electromagnetic process is clearly shown in Fig. 6, where a plot of the asymmetry $\Sigma\left(\theta_{\mathrm{n}}=\pi / 2\right)$ vs the laboratory $\gamma$-ray energy is given. ${ }^{42}$ Our data are compared with the results of earlier experiments ${ }^{39,41,43,44}$ and with the theoretical RSC calculation carried out by Arenhövel. ${ }^{10}$ The dashed line ( $N=$ normal) has been obtained in the standard Partovi approximation; the solid line $(N+$ MEC + IC) contains MEC + IC corrections to the current operator. Moreover, as shown in Ref. 13, relativistic charge corrections may also sensibly affect the theoretical curves at the highest energies.

In Figs. 7(a)-(d) our data are compared with those of other authors. Figure 7(a) shows our data at $19.8 \mathrm{MeV}$ in comparison with the $20 \mathrm{MeV}$ results of Halpern and Weinstock $^{50}$ and Shin et al. ${ }^{51}$ In Fig. 7(b) the $30 \mathrm{MeV}$ data of Shin ${ }^{51}$ and Weissman and Shultz ${ }^{33}$ are reported, together with Allen ${ }^{52}$ results interpolated for $29.0 \mathrm{MeV}$; Fig. 7 (c) shows the $40 \mathrm{MeV}$ results of Refs. 51 and 33 . 
Finally, Fig. 7(b) displays the $60 \mathrm{MeV}$ data obtained by Galey $^{53}$ and the data of Shin ${ }^{51}$ interpolated to $60.8 \mathrm{MeV}$. As can be readily checked, these data are in substantial agreement.

A standard least-squares procedure was used to fit the experimental points, expanding $I_{0}(\theta) / \sigma_{t}$ with the sum of Legendre polynomials $P_{i}(\cos \theta)$, and $I_{1}(\theta) / \sigma_{t}$ with the sum of associated Legendre functions $P_{i}^{2}(\cos \theta)$, as shown in Eqs. (4).

The results obtained for the coefficients $A_{i} / \sigma_{t}$ ( $i=1,2,3,4)$ are displayed in Fig. 8 vs the laboratory $\gamma$ ray energy, together with the results obtained from a reexamination of the earlier measurements. The error bars shown are derived from the least-squares procedure.

Theoretical curves obtained in Refs. 5 and 10 and Refs. 11 and 16 with the RSC and DTS-B potentials, including $\mathrm{MEC}+\mathrm{IC}$ effects in the Siegert approximation, are also shown in Fig. 8. The ratio $A_{1} / \sigma_{t}$ seems to be consistent with the theory, while the value of $A_{2} / \sigma_{t}$ displays a significant difference at $E_{\gamma} \geq 50 \mathrm{MeV}$. This result has already been pointed out in Ref. 35. Preliminary estimates $^{11}$ of the relativistic and pion corrections to the charge density operator seem to enhance $\left|A_{2}\right|\left(A_{2}<0\right)$ by a substantial amount. Similar arguments hold for $A_{3}$, but here the situation appears to be less definite, owing to the size of the quoted errors.

The results obtained for the coefficients $B_{i} / \sigma_{t}$ $(i=2,3,4)$ are reported in Fig. 9, together with the RSC and DTS-B theoretical predictions.

Additional information that can shed some more light on the details of the problem may be obtained from the coefficients $a, \ldots, h$ of the angular distributions formulation introduced by Partovi ${ }^{49}$ [see Eqs. (5)].

Least-square fitted coefficients $a / \sigma_{t}, \ldots, e / \sigma_{t}$ are compared directly with their predicted values in Fig. 10. At $E_{\gamma}=60.8 \mathrm{MeV}$, the coefficient $c$ remained completely undetermined and had to be fixed at its theoretical value (the cross in Fig. 10). In this case only the determination of $d$ is sensibly affected by the assumed $c$ value. Our estimate (Fig. 12) of $I_{0}(\theta=0)=a+c$ (obtained by multiplying our results for the $\sigma_{t}$ estimates of Ref. 35) substantially agree with the corresponding Mainz, ${ }^{36,57}$ Louvain, ${ }^{37,58}$ and Indiana ${ }^{38}$ results, even if some indications could exist for a trend of the data slightly higher than the one pictured by the Mainz and Louvain values only. Since the consistency among all these results is only limited within an accuracy of $\sim 20 \%$, there is still a need for new independent measurement of $a$ (and $c$ ), that is, of $I_{0}(\theta=0)$ and $I_{0}(\theta=\pi)$. This point will be discussed further in the next section. Also, the disagreement observed for $d / \sigma_{t}$ must be further explored, both on the theoretical and the experimental side.

Our experimental estimates for the $f / \sigma_{t}, \ldots, h / \sigma_{t}$ coefficients are reported in Fig. 11 together with the corresponding theoretical RSC and DTS-B estimates.

A summary of all coefficients obtained in our fits is shown in Tables V and VI. Only statistical errors are appended.

\section{CONCLUSIONS}

The bulk of the existing data (see, i.e., Fig. 6) clearly shows the role of the non-nucleonic degrees of freedom in the deuteron photodisintegration. Moreover, the measured values of the $A_{i} / \sigma_{t}, B_{i} / \sigma_{t}$, and $a / \sigma_{t}, \ldots, h / \sigma_{t}$ coefficients lie generally in the theoretically predicted ranges, with a few exceptions.

On the other hand, all theoretical predictions at higher energies could be appreciably modified by the inclusion of relativistic and two-body exchange contributions to the charge density operator. In addition, the two-body charge effects, particularly as far as the $a$ and $c$ coefficients are concerned, are markedly different if pseudoscalar (PS) or pseudovector (PV) coupling is assumed. ${ }^{10,15,16}$ Thus a definitive analysis of the data presently on hand must be postponed until extensive theoretical calculations for different $\mathrm{N}-\mathrm{N}$ potentials become available. In particular, one can hope that a careful examination of the $a$ and $c$ coefficients (and perhaps of the other ones) will permit an unequivocal choice between PS and PV couplings and a new determination of the $D$-wave asymptotic $A_{D}$ amplitude, ${ }^{54}$ strictly connected to the deuteron $D$-state percentage $P_{D}$.

Our final remarks concern the $a, \ldots, h$ coefficients. Their extraction from the experimental data is considerably more uncertain than the determination of the $A_{i}$ and $B_{i}$ coefficients, because in the fitting procedure they appear to be strongly correlated. Keeping this point in mind, let us further recall that our estimates of $(a+c) / \sigma_{t}$ seem to be only slightly higher than the corresponding Mainz results, ${ }^{36}$ normalized by assuming standard $\sigma_{t}$ values. ${ }^{35}$ Recent calculations including relativistic and two-body charge contributions ${ }^{11,15,16}$ seem to suggest that our results can be partially reproduced by assuming a PV coupling and using potentials that give $P_{D}$ percentages in the range $(6-8) \%$. However, our estimates of $a / \sigma_{t}$ and of $c / \sigma_{t}$ are rather indirect and must be tested by further direct measurements of $I_{0}(\theta=0)$ and $I_{0}(\theta=\pi)$.

As a concluding remark, let us recall that besides the well-known dependence on the standard parameters characterizing the $\mathrm{N}-\mathrm{N}$ potentials, the deuteron photodisintegration angular distributions $I_{0}(\theta)$ and $I_{1}(\theta)$ could be explicitly affected by the internal nucleonic degrees of freedom, that is, by their internal quark structure. To the best of our knowledge, only the influence of the nucleon structure on the total photodisintegration cross section $\sigma_{t}$ has been so far studied, and in a preliminary way ${ }^{55}$ at that. This point too must be investigated further before any definitive conclusions on the role of non-nucleonic degrees of freedom in deuteron photodisintegration may be extracted from our data.

\section{ACKNOWLEDGMENTS}

We wish to thank the technical staff of the LADON Group, E. Cima, A. Dante, L. Ruggieri, and E. Turri, for their dedicated help throughout this experiment. The expert operation of the storage ring by the Adone staff is warmly acknowledged. 
${ }^{1}$ M. L. Rustgi, O. P. Rustgi, and T. S. Sandhu, Can. J. Phys. 55, 158 (1977).

2J. M. Laget, Nucl. Phys. A312, 265 (1978).

${ }^{3} \mathrm{H}$. Arenhövel, in Few Body Systems and Electromagnetic Interactions, Vol. 86 of Lecture Notes in Physics, edited by C. Ciofi degli Atti and E. De Sanctis (Springer, New York, 1978).

${ }^{4}$ K. Ogawa, T. Kamae, and K. Nakamura, Nucl. Phys. A340, 451 (1980).

${ }^{5}$ H. Arenhövel, Z. Phys. A 302, 25 (1981).

${ }^{6}$ M. Anastasio and M. Chemtob, Nucl. Phys. A364, 219 (1981).

${ }^{7}$ A. Cambi, B. Mosconi, and P. Ricci, Phys. Rev. C 23, 992 (1981).

${ }^{8}$ Reeta Vyas, Manoj Chopra, and M. L. Rustgi, Phys. Rev. C 25, 1801 (1982).

${ }^{9}$ Reeta Vyas and M. L. Rustgi, Phys. Rev. C 26, 1399 (1982).

${ }^{10}$ H. Arenhövel, Nucl. Phys. A374, 521c (1982); Nuovo Cimento 76 A, 256 (1983); contribution to the Workshop on Perspectives in Nuclear Physics at Intermediate Energies, Trieste, 1983; private communication.

${ }^{11}$ A. Cambi, B. Mosconi, and P. Ricci, Phys. Rev. C 26, 2358 (1982); J. Phys. G 10, L11 (1984); private communication.

${ }^{12}$ M. L. Rustgi, Reeta Vyas, and Manoj Chopra, Phys. Rev. Lett. 50, 236 (1983).

${ }^{13}$ M. L. Rustgi and Reeta Vyas, Phys. Lett. 121B, 365 (1983).

${ }^{14}$ M. L. Rustgi, R. D. Nanemaket, and R. D. Sharma, Nuovo Cimento 77 A, 317 (1983).

15J. L. Friar, B. F. Gibson, and G. L. Payne, Phys. Rev. C 30, 441 (1984).

${ }^{16}$ A. Cambi, B. Mosconi, and P. Ricci, contribution to the Workshop on Perspectives in Nuclear Physics at Intermediate Energies, Trieste, 1983; private communication.

${ }^{17}$ W. Y. P. Hwang, J. T. Londergan, and G. E. Walker, Ann. Phys. (N.Y.) 149, 335 (1983).

${ }^{18}$ M. L. Rustgi, Reeta Vyas, and O. P. Rustgi, Phys. Rev. C 29, 785 (1984).

${ }^{19}$ M. L. Rustgi, T. S. Sandhu, and O. P. Rustgi, Phys. Lett. 70B, 145 (1977); Phys. Rev. C 20, 24 (1979).

${ }^{20}$ E. L. Lomon, Phys. Lett. 68B, 419 (1977).

${ }^{21}$ M. Gari and B. Sommer, Phys. Rev. Lett. 41, 22 (1978).

${ }^{22}$ E. Hadjimichael, Phys. Lett. 85B, 17 (1979).

${ }^{23}$ H. Arenhövel and W. Fabian, Phys. Rev. Lett. 47, 34 (1981).

${ }^{24}$ W. Jaus and W. S. Woolkock, Nucl. Phys. A365, 477 (1981).

${ }^{25}$ A. Cambi, B. Mosconi, and P. Ricci, Phys. Rev. Lett. 48, 462 (1982).

${ }^{26}$ L. Federici, G. Giordano, G. Matone, G. Pasquariello, P. G. Picozza, R. Caloi, L. Casano, M. P. De Pascale, M. Mattioli, E. Poldi, C. Schaerf, M. Vanni, P. Pelfer, D. Prosperi, S. Frullani, and B. Girolami, Nuovo Cimento B 59, 247 (1980).

${ }^{27}$ M. P. De Pascale, G. Giordano, G. Matone, P. Picozza, R. Caloi, L. Casano, M. Mattioli, E. Poldi, D. Prosperi, and C. Schaerf, Appl. Phys. B28, 151 (1982).

${ }^{28}$ O. F. Kulikov, Y. Y. Telnov, E. I. Filippov, and M. N. Yakimenko, Phys. Lett. 13, 344 (1964).

${ }^{29}$ C. Bemporad, R. H. Milburn, N. Tanaka, and M. Fotino, Phys. Rev. 138, 1546 (1965)

${ }^{30}$ J. Ballam, G. B. Chadwick, R. Gearhart, Z. G. T. Guiragossian, P. R. Klein, A. Levy, M. Menke, J. J. Murray, P. Seyboth, G. Wolf, C. K. Sinclair, H. H. Bingham, W. B. Fretter, K. C. Moffeit, W. J. Podolsky, M. S. Rabin, A. H. Rosenfeld, and R. Windmolders, Phys. Rev. Lett. 23, 498 (1969).

${ }^{31}$ G. Matone and A. Tranquilli, Laboratori Nazionali di Frascati Report LNF-75/21, 1975.

${ }^{32}$ M. P. De Pascale, G. Giordano, G. Matone, P. Picozza, R. Caloi, L. Casano, M. Mattioli, E. Poldi, D. Prosperi, and C. Schaerf, Appl. Opt. 21, 2660 (1982).

${ }^{33}$ B. Weissman and H. L. Schultz, Nucl. Phys. A174, 129 (1971).

34J. E. E. Baglin, R. W. Carr, E. J. Bentz, Jr., and C. P. Wu, Nucl. Phys. A201, 593 (1973).

${ }^{35}$ M. P. De Pascale, G. Giordano, G. Matone, P. Picozza, L. Azario, R. Caloi, L. Casano, L. Ingrosso, M. Mattioli, E. Poldi, D. Prosperi, and C. Schaerf, Phys. Lett. 119B, 30 (1982).

${ }^{36}$ R. Hughes, A. Zieger, H. Wäffler, and B. Ziegler, Nucl. Phys. A267, 329 (1976).

37J. F. Gilot, A. Bol, P. Leleux, P. Lipnik, and P. Macq, Phys. Rev. Lett. 47, 304 (1984).

${ }^{38}$ H. O. Meyer, J. R. Hall, H. Hugi, H. J. Karwowski, R. E. Pollock, and P. Schwandt, Phys. Rev. Lett. 52, 1759 (1984).

${ }^{39}$ F. F. Liu, Phys. Rev. 138, B1443 (1965).

${ }^{40} \mathrm{G}$. Barbiellini, C. Bernardini, F. Felicetti, and G. P. Murtas, Phys. Rev. 154, 988 (1967).

${ }^{41}$ W. Del Bianco, H. Jeremie, M. Irshad, and G. Kajrys, Nucl. Phys. A343, 121 (1980).

${ }^{42}$ W. Del Bianco, L. Federici, G. Giordano, G. Matone, G. Pasquariello, P. Picozza, R. Caloi, L. Casano, M. P. Dé Pascale, L. Ingrosso, M. Mattioli, E. Poldi, C. Schaerf, P. Pelfer, D. Prosperi, S. Frullani, B. Girolami, and H. Jeremie, Phys. Rev. Lett. 47, 1118 (1981).

${ }^{43}$ M. P. De Pascale, L. Federici, G. Giordano, G. Matone, P. Picozza, L. Azario, R. Caloi, L. Casano, M. Mattioli, E. Poldi, D. Prosperi, and C. Schaerf, Phys. Lett. 114B, 11 (1982).

${ }^{44}$ V. G. Gorbenko, Yu. V. Zhebrovskij, L. Ya. Kolesnikov, A. L. Rubashkin, and P. V. Sorokin, Nucl. Phys. A381, 330 (1982).

${ }^{45}$ R. V. Reid, Jr., Ann. Phys. (N.Y.) 50, 411 (1968).

${ }^{46}$ T. Hamada and J. D. Johnston, Nucl. Phys. B34, 382 (1962).

${ }^{47}$ M. Lacombe, B. Loiseau, J. M. Richard, R. Vinh Mau, J. Côté, P. Pirès, and R. De Tourreil, Phys. Rev. C 21, 861 (1980); 23, 2405 (1981).

${ }^{48}$ R. De Tourreil and D. W. L. Sprung, Nucl. Phys. A201, 193 (1973).

${ }^{49}$ F. Partovi, Ann. Phys. (N.Y.) 27, 79 (1964).

50J. Halpern and E. V. Weinstock, Phys. Rev. 91, 934 (1953).

51Y. M. Shin, J. A. Rawlins, W. Buss, and A. O. Evwaraye, Nucl. Phys. A154, 482 (1970).

${ }^{52}$ L. Allen, Jr., Phys. Rev. 98, 705 (1955).

53J. A. Galey, Phys. Rev. 117, 763 (1960).

${ }^{54}$ M. E. Schulze, D. P. Saylor, and R. Goloskie, Phys. Rev. C 24, 2435 (1981).

${ }^{55}$ E. Hadjimichael and D. P. Saylor, Phys. Rev. Lett. 45, 1776 (1980).

${ }^{56}$ Yu. A. A. Aleksandrov, N. B. Delone, L. I. Slovokhotov, G. A. Sokol, and L. N. Shtarkov, Zh. Eksp. Teor. Fiz. 33, 614 (1957) [Sov. Phys.-JETP 6, 472 (1958)].

${ }^{57}$ A. Zieger, P. Grewer, and B. Ziegler, Abstract of 11th Europhysics Divisional Conference-Nuclear Physics with Electromagnetic Probes, Paris, 1985, edited by European Physical Society, Vol. C17, 1985, p. 140.

${ }^{58}$ A. Ninane, C. Dupont, P. Leleux, P. Lipnik, and P. Macq, Can. J. Phys. 62, 1104 (1984) 